\title{
A NOTE ON THE NUMERICAL APPROACH FOR THE REACTION-DIFFUSION PROBLEM WITH A FREE BOUNDARY CONDITION
}

\author{
E. ÖZUĞURLU ${ }^{1}$
}

(Received 22 May, 2009; revised 24 March, 2010)

\begin{abstract}
The equation modelling the evolution of a foam (a complex porous medium consisting of a set of gas bubbles surrounded by liquid films) is solved numerically. This model is described by the reaction-diffusion differential equation with a free boundary. Two numerical methods, namely the fixed-point and the averaging in time and forward differences in space (the Crank-Nicolson scheme), both in combination with Newton's method, are proposed for solving the governing equations. The solution of Burgers' equation is considered as a special case. We present the Crank-Nicolson scheme combined with Newton's method for the reaction-diffusion differential equation appearing in a foam breaking phenomenon.
\end{abstract}

2000 Mathematics subject classification: primary 35K55; secondary 35R35, 65M06, 65M12, 76B45, 76D99.

Keywords and phrases: foam drainage, nonlinear partial differential equation, CrankNicolson method, breaking front, Plateau border, reaction-diffusion problem, free boundary problem.

\section{Introduction}

Foam drainage is a complex physico-chemical hydrodynamic process, as defined and explored in Kruglyakov et al. [16]. It is an important application area in industry since many technological properties of foam depend on the liquid volume fraction and the drainage rate. It is therefore important to understand how a foam forms and how it evolves in time.

Drainage is defined as the transport of liquid through foam driven by gravity or pressure differences. The Plateau border (named after a blind Belgian scientist), which is a transition zone separating bulk surfaces, frames or other films from the film proper and always containing some bulk liquid, plays an important role in foam drainage studies.

\footnotetext{
${ }^{1}$ Bahçeşehir University, Faculty of Arts and Sciences, Department of Mathematics and Computer Sciences, 34353 Beşiktaş, Istanbul, Turkey; e-mail: ersin.ozugurlu@ bahcesehir.edu.tr.

(C) Australian Mathematical Society 2010, Serial-fee code 1446-1811/2010 \$16.00
} 
Three factors, namely, the liquid distribution between the Plateau borders, the liquid films between two foam bubbles, and the distribution along the foam height, have to be known for the design of some technological processes; see, for example, Banhart and Weaire [2], Exerowa and Kruglyakov [11] and Weaire [19].

In Exerowa and Kruglyakov [11], foam and foam films are considered, giving special attention to stability and drainage of foams. Experimental and theoretical aspects of the foam drainage equation are also considered in Koehler et al. [13], where an energy argument takes into account viscous dissipation in both the Plateau border and nodes to generalize the foam drainage equation. Three types of experiments, namely forced, free and pulsed drainage, are investigated.

The numerical solution of the foam drainage equation has been studied by many researchers, including Colin and Fabrie [4, 5], Cox et al. [6], Durand and Langevin [10], Koehler et al. [14], Verbist et al. [18] and Weaire et al. [22]. In 2003, Weaire et al. [22] looked at problems concerning the fluid dynamics of foam under drainage, that is, the transport of liquid through foam. That the flow of liquid through a foam occurs mainly in its Plateau borders is shown using the surface evolver program (see Brakke [3]). An expanded review focusing on recent works on foam drainage including both the advanced theoretical and experimental studies of the foam drainage equation is given in Kruglyakov et al. [16]. Recently, Dahmani et al. [8] solved the foam drainage equation with time-fractional and space-fractional derivatives by applying the Adomian decomposition method.

Here, however, we are interested in the case where the foam breaks due to the rupture of thin films during the drying process (see Colin and Fabrie [5], where the theoretical aspects of the convergence and well-posedness of the foam drainage equation are discussed).

\section{Formulation of the problem}

The foam drainage equation is a nonlinear partial differential equation that describes the evolution of the vertical density profile of foam under gravity. It was derived in 1965 by Leonard and Lemlich [17] on the basis of various simplifying assumptions regarding the nature of this drainage process. The attribution of drainage entirely to Poiseuille flow in the network of Plateau borders is very important. These are the liquid-filled interstices of the bubbles which pack together to form the foam.

Most of the essentials of the foam theory are to be found in Leonard and Lemlich [17]. In Gol'dfarb et al. [12], the following foam drainage equation is derived from the equation of continuity in the dimensional form, treating the liquid as incompressible for a single vertical Plateau border (channel-dominated) with crosssection $A(\tau, \xi)$, which depends on the downward vertical coordinate $\xi$ and time $\tau$,

$$
\frac{\partial}{\partial \tau} A(\tau, \xi)+\frac{\partial}{\partial \xi}[A(\tau, \xi) q(\tau, \xi)]=0
$$

where the velocity $q$ is averaged over the cross-section of the Plateau border. Gol'dfarb et al. [12] assumed that the flow in the channels formed by the Plateau 
borders is of the Poiseuille type, that is, with zero velocity at the boundaries. In particular, the assumption of Poiseuille flow requires a sufficient surface viscosity to render the surfaces of the Plateau borders effectively rigid; see Kraynik [15] for details. Disregarding the film flow may also depend, to some extent, on this assumption; see Verbist et al. [18]. We also assume that drainage is sufficiently rapid that the diffusion of gas between cells is negligible. This is the process that leads to foam coarsening over long times. We neglect inertial effects and assume that there is a simple Poiseuille-type dissipation which is proportional to the mean liquid velocity in the Plateau border and inversely proportional to the cross section area.

The following foam drainage equation is derived from assumptions which best apply to dry foam. The contribution of the films to drainage is not included in this model. On the basis of simple considerations of continuity and pressure balance, as well as the treatment of dissipation in a manner analogous to Darcy's law in the theory of porous media, we follow the steps given in Weaire and Hutzler [21] to write the above Equation (2.1) in dimensionless variables,

where

$$
\frac{\partial u}{\partial t}+\frac{\partial}{\partial x}\left(u^{2}-\varepsilon \sqrt{u} \frac{\partial u}{\partial x}\right)=0
$$

$$
\varepsilon=\frac{v M}{2 \pi \rho g \sqrt{\pi A_{i}}}
$$

and where $M=(\sqrt{3}-\pi / 2)^{1 / 2}$ is a geometrical constant corresponding to the triangular form of the Plateau border cross section and $A_{i}$ is a typical value of the area of the Plateau borders. The physical parameters are $v$ (constant surface tension), not including any effects of surface elasticity, $\rho$ (liquid density), $g$ (acceleration due to gravity), and $\eta^{*}$ (effective viscosity). (For more details see the appendix in Verbist et al. [18].) In Equation (2.2), $u(t, x)$ is the dimensionless cross section area of Plateau borders at time $t$ and level $x$, that is, $u(t, x)$ is a local measure of the foam's liquid. Here, dimensionless coordinates, corresponding to vertical position $x$ (measured downwards) and time $t$, are introduced by defining $x=\xi / x_{0}$ and $t=\tau / t_{0}$ where the units are $x_{0}=(C v / \rho g)^{1 / 2}$ and $t_{0}=\eta^{*} /(C v \rho g)^{1 / 2}$, and $u(t, x)=A(t, x) / x_{0}^{2}$.

Note that $x_{0}^{2}$ is (to within a constant) the capillary constant which has commonly been used in the theory of surfaces, and the height of meniscus is a useful indication of the magnitude of $x_{0}$. The constant $t_{0}$, which sets the time-scale, is less familiar but is proportional to viscosity.

The solution of (2.2) describes (for fixed $t$ ) a snapshot of the vertical density profile of the foam. The term in brackets in (2.2) is the dimensionless flow rate

$$
Q=u^{2}-\varepsilon \sqrt{u} \frac{\partial u}{\partial x} \text {. }
$$

For a foam in equilibrium, we set $Q=0$, which gives an equilibrium profile

$$
u_{e q}(x)=\left(\frac{1}{\sqrt{u_{R}}}+\frac{R-x}{2 \varepsilon}\right)^{-2}
$$


where $u_{R}$ is the liquid fraction at the bottom of the foam (that of a bubbly liquid at the rigidity loss transition where the bubbles of the foam separate) and $R$ is the height of the foam. Notice that $u_{R}$ is never zero.

A trivial solution of (2.2) is that of steady drainage with constant flow rate. Here $u=u_{0}$, a constant, and so the flow rate is $u_{0}^{2}$ and the flow velocity is $u_{0}$.

Equation (2.2) was called the foam drainage equation by Verbist et al. [18] and has to be completed with boundary conditions. In the case where the foam is stable during the draining (that is, does not break) we take $Q=0$ at $x=0$, and thus

$$
u^{2}-\varepsilon \sqrt{u} \frac{\partial u}{\partial x}=0
$$

which means that no water enters the foam at the top level. The boundary condition at the bottom is more difficult to define. One reason for this is the assumption of dry foam while in reality the foam is wet at its bottom extremity where it touches the liquid. Here, we accept the assumptions that were suggested in Colin and Fabrie [5], where the problem posed on the half line was considered. Equation (2.2), associated with the above boundary condition (2.3), was considered in Koehler et al. [14]; we do not present any mathematical study of it, but draw attention to Koehler et al. [14] for numerical and experimental results.

The situation where the foam breaks during the drying process is very important. In other words, at time $t$, the foam is confined in the domain $[\gamma(t),+\infty)$, where $\gamma(t)$ denotes the position of the breaking front. In a physical sense, this means that the foam breaks when the area of the Plateau borders reaches a critical value $\beta>0$. Therefore, the boundary condition at $x=\gamma(t)$ is $u(t, \gamma(t))=\beta$.

So far, the case where $d \gamma(t) / d t=0$ has been examined. See Colin and Fabrie [5] for details, and the references therein.

We consider the case where $d \gamma(t) / d t \neq 0$, which is extremely important and completely different from the cases considered up to now. It is known that conservation of the quantity of water at the breaking front leads us to

$$
\frac{d \gamma(t)}{d t}=\beta-\frac{\varepsilon}{\sqrt{\beta}}\left[\frac{\partial u(t, x)}{\partial x}\right]_{x=\gamma(t)} .
$$

Therefore, the problem we have to deal with reads

$$
\frac{\partial u}{\partial t}+\frac{\partial u^{2}}{\partial x}-\varepsilon \frac{\partial}{\partial x}\left(\sqrt{u} \frac{\partial u}{\partial x}\right)=0,
$$

with the boundary conditions

$$
\begin{aligned}
u(t, \gamma(t)) & =\beta, \\
\dot{\gamma}(t) & =\beta-\frac{\varepsilon}{\sqrt{\beta}}\left[\frac{\partial u(t, x)}{\partial x}\right]_{x=\gamma(t)}
\end{aligned}
$$


and the initial condition

$$
u(0, x)=u_{0}(x), \quad x \in(\gamma(t),+\infty)
$$

where $0<\beta<1$ and $u_{0}(x) \geq \beta, x \in(\gamma(0),+\infty)$. In order to study (2.5) we make a transformation $v(t, x)=u(t, x+\gamma(t))$. Then the equation satisfied by $v$ is

$$
\frac{\partial v}{\partial t}-\dot{\gamma}(t) \frac{\partial v}{\partial x}+\frac{\partial v^{2}}{\partial x}-\varepsilon \frac{\partial}{\partial x}\left(\sqrt{v} \frac{\partial v}{\partial x}\right)=0,
$$

with the boundary conditions

$$
\begin{aligned}
v(t, 0) & =\beta, \\
\dot{\gamma}(t) & =\beta-\frac{\varepsilon}{\sqrt{\beta}}\left[\frac{\partial v(t, x)}{\partial x}\right]_{x=0}, \quad \gamma(0)=0,
\end{aligned}
$$

and the initial conditions

$$
\begin{gathered}
\gamma(0)=0, \\
v(0, x)=v_{0}(x), \quad x \in(0,+\infty) .
\end{gathered}
$$

We consider the bounded interval $(0, R)$ with homogeneous Neumann boundary condition at $x=R$. Hence, the problem becomes

$$
\frac{\partial v}{\partial t}-\dot{\gamma}(t) \frac{\partial v}{\partial x}+\frac{\partial v^{2}}{\partial x}-\varepsilon \frac{\partial}{\partial x}\left(\sqrt{v} \frac{\partial v}{\partial x}\right)=0, \quad t>0,
$$

with the boundary conditions

$$
\begin{aligned}
v(t, 0) & =\beta, \quad\left[\frac{\partial v(t, x)}{\partial x}\right]_{x=R}=0, \quad t \geq 0, \\
\dot{\gamma}(t) & =\beta-\frac{\varepsilon}{\sqrt{\beta}}\left[\frac{\partial v(t, x)}{\partial x}\right]_{x=0}, \quad \gamma(0)=0, \quad t \geq 0
\end{aligned}
$$

and the initial conditions

$$
\begin{gathered}
\gamma(0)=0, \\
v(0, x)=v_{0}(x), \quad x \in(0, R)
\end{gathered}
$$

where $R$ is the height of the foam and an arbitrary but fixed positive number. Colin and Fabrie [5] considered Equation (2.6) and assumed that

$$
\begin{gathered}
0<\beta<1, \\
v_{0} \in W^{1, \infty}(0, R), \quad v_{0}(0)=\beta, \\
v_{0}(x) \geq \beta, \quad x \in(0, R)
\end{gathered}
$$

where $W^{1, \infty}$ is the usual Sobolev space; see Adams [1]. Colin and Fabrie [5] constructed the global solutions of (2.6). 
We consider the equation governing the evolution of a foam Equation (2.6) with the above boundary and initial conditions. Cox et al. [7] considered the case of $\dot{\gamma}(t)=0$ using a constant-step-size finite-difference representation, with forward differencing in time (upwind) and space.

\section{Numerical procedure}

We assume that $v(t, x) \geq 0$, given by (2.6), since $v(t, x)$ represents the dimensionless cross section area of Plateau borders at time $t$ and level $x$.

Therefore, we use the transformation $z(t, x)=\left[v(t, x)^{3 / 2}\right]$. Hence, Equation (2.6) becomes

$$
\frac{2}{3} \frac{1}{z^{1 / 3}} \frac{\partial z}{\partial t}-\frac{2}{3} \frac{1}{z^{1 / 3}} \frac{\partial z}{\partial x}\left(\beta-\frac{2 \varepsilon}{3 \beta}\left[\frac{\partial z(t, x)}{\partial x}\right]_{x=0}\right)+\frac{4}{3} z^{1 / 3} \frac{\partial z}{\partial x}-\frac{2}{3} \varepsilon \frac{\partial^{2} z}{\partial x^{2}}=0
$$

with the boundary conditions

$$
\begin{aligned}
& z(t, 0)=\beta^{3 / 2}, \quad\left[\frac{\partial z(t, x)}{\partial x}\right]_{x=R}=0, \quad t \geq 0, \\
& \dot{\gamma}(t)=\beta-\frac{\varepsilon}{\sqrt{\beta}}\left[\frac{2}{3} \frac{1}{z^{1 / 3}} \frac{\partial z(t, x)}{\partial x}\right]_{x=0}, \quad t \geq 0
\end{aligned}
$$

and initial conditions

$$
\begin{gathered}
\gamma(0)=0, \quad t \geq 0, \\
z(0, x)=z_{0}(x), \quad x \in(0, R) .
\end{gathered}
$$

Equation (3.1) is multiplied by $\frac{3}{2} z^{1 / 3}$, giving

$$
\frac{\partial z}{\partial t}-\frac{\partial z}{\partial x}\left(\beta-\frac{2 \varepsilon}{3 \beta}\left[\frac{\partial z(t, x)}{\partial x}\right]_{x=0}\right)+2 z^{2 / 3} \frac{\partial z}{\partial x}-\varepsilon z^{1 / 3} \frac{\partial^{2} z}{\partial x^{2}}=0 .
$$

3.1. Fixed-point iteration method The partial differential equation (3.3) is discretized in space by standard finite differences on an equally spaced grid and using a forward-time difference scheme with constant step size. The implicit scheme then becomes

$$
\begin{aligned}
& \left(\frac{z_{m}^{n+1}-z_{m}^{n}}{\Delta t}\right)-\left(\frac{z_{m+1}^{n+1}-z_{m}^{n+1}}{\triangle x}\right)\left(\beta-\frac{2 \varepsilon}{3 \beta}\left(\frac{z_{1}^{n+1}-z_{0}^{n+1}}{\triangle x}\right)\right) \\
& +2\left(z_{m}^{n+1}\right)^{2 / 3}\left(\frac{z_{m+1}^{n+1}-z_{m}^{n+1}}{\triangle x}\right) \\
& -\varepsilon\left(z_{m}^{n+1}\right)^{1 / 3}\left(\frac{z_{m+1}^{n+1}-2 z_{m}^{n+1}+z_{m-1}^{n+1}}{(\triangle x)^{2}}\right)=0
\end{aligned}
$$

where $z_{m}^{n}=z\left(t_{n}, x_{m}\right)=z(n k, m h), k=\Delta t, h=\Delta x$ and $m=0, \ldots, M-1$. We refer to this method as Method $I$. 
Equation (3.4) becomes

$$
\begin{aligned}
z_{m}^{n+1}=z_{m}^{n} & +\Delta t\left(\frac{z_{m+1}^{n+1}-z_{m}^{n+1}}{\Delta x}\right)\left(\beta-\frac{2 \varepsilon}{3 \beta}\left(\frac{z_{1}^{n+1}-z_{0}^{n+1}}{\triangle x}\right)\right) \\
& -2 \triangle t\left(z_{m}^{n+1}\right)^{2 / 3}\left(\frac{z_{m+1}^{n+1}-z_{m}^{n+1}}{\triangle x}\right) \\
& +\varepsilon \triangle t\left(z_{m}^{n+1}\right)^{1 / 3}\left(\frac{z_{m+1}^{n+1}-2 z_{m}^{n+1}+z_{m-1}^{n+1}}{(\triangle x)^{2}}\right) .
\end{aligned}
$$

Fixed point iteration is applied to (3.5) in time using

$$
\begin{aligned}
z_{m}^{n+1, k+1}=z_{m}^{n, k} & +\Delta t\left(\frac{z_{m+1}^{n+1, k}-z_{m}^{n+1, k}}{\triangle x}\right)\left(\beta-\frac{2 \varepsilon}{3 \beta}\left(\frac{z_{1}^{n+1, k}-z_{0}^{n+1, k}}{\triangle x}\right)\right) \\
& -2 \triangle t\left(z_{m}^{n+1, k}\right)^{2 / 3}\left(\frac{z_{m+1}^{n+1, k}-z_{m}^{n+1, k}}{\triangle x}\right) \\
& +\varepsilon \triangle t\left(z_{m}^{n+1, k}\right)^{1 / 3}\left(\frac{z_{m+1}^{n+1, k}-2 z_{m}^{n+1, k}+z_{m-1}^{n+1, k}}{(\triangle x)^{2}}\right)
\end{aligned}
$$

which is written in more compact form as

$$
z_{m}^{n+1, k+1}=z_{m}^{n, k}+F\left(z_{m+1}^{n+1, k}, z_{m}^{n+1, k}, z_{m-1}^{n+1, k}\right) .
$$

This fixed point iteration method is first-order accurate. Thus, this implicit method (Method I) is only first-order accurate in time and second-order accurate in space.

3.2. The Crank-Nicolson scheme The Crank-Nicolson scheme is applied to (3.3), yielding

$$
\begin{aligned}
& \left(\frac{z_{m}^{n+1}-z_{m}^{n}}{\triangle t}\right)-\frac{1}{2}\left[\left(\frac{z_{m+1}^{n+1}-z_{m}^{n+1}}{\triangle x}\right)+\left(\frac{z_{m+1}^{n}-z_{m}^{n}}{\Delta x}\right)\right] \\
& \quad \times\left[\beta-\frac{2 \varepsilon}{3 \beta} \frac{1}{2}\left(\frac{z_{1}^{n+1}-z_{0}^{n+1}}{\Delta x}+\frac{z_{1}^{n}-z_{0}^{n}}{\triangle x}\right)\right] \\
& \quad+\left(\frac{z_{m}^{n+1}+z_{m}^{n}}{2}\right)^{2 / 3}\left(\frac{z_{m+1}^{n+1}-z_{m}^{n+1}}{\triangle x}+\frac{z_{m+1}^{n}-z_{m}^{n}}{\triangle x}\right)-\frac{\varepsilon}{2}\left(\frac{z_{m}^{n+1}+z_{m}^{n}}{2}\right)^{1 / 3} \\
& \quad \times\left(\frac{z_{m+1}^{n+1}-2 z_{m}^{n+1}+z_{m-1}^{n+1}}{(\triangle x)^{2}}+\frac{z_{m+1}^{n}-2 z_{m}^{n}+z_{m-1}^{n}}{(\triangle x)^{2}}\right)=0 .
\end{aligned}
$$

We define the $M$ mesh points $x_{I}=(I-1) E /\left(2^{1 / 3}\right), I=1, \ldots, M$ where $E=0.11$ and the interval of discretization is chosen arbitrarily. The $z_{m}^{n+1}$ values are 
TABLE 1. The range of the parameters for each simulation.

\begin{tabular}{llllll}
\hline Figure & $\varepsilon$ & $\beta$ & $\Delta x$ & $\Delta t$ & Method \\
\hline 1 & 0.01 & 0.001 & 0.0873 & 0.0873 & Crank-Nicolson \\
2 & 0.01 & 0.001 & 0.0873 & 0.1 & Implicit (Method I) \\
5 & 0.01 & 0.99 & 0.0873 & 0.0873 & Crank-Nicolson \\
6 & 0.01 & 0.99 & 0.0873 & 0.0873 & Implicit (Method I) \\
7 & $10^{-7}$ & 0.001 & 0.0873 & 0.0873 & Crank-Nicolson \\
8 & $10^{-7}$ & 0.99 & 0.0873 & 0.0873 & Crank-Nicolson \\
\hline
\end{tabular}

unknowns for $m=0, \ldots, M-1$. For $m=0$, we use the forward difference formula; for $m=M-1$, we impose the boundary condition $[\partial z(t, x) / \partial x]_{x=R}=0$ using the backward second-order difference formula $z_{M}^{n}=4 / 3 z_{M-1}^{n}-1 / 3 z_{M-2}^{n}$.

In both Method I and the Crank-Nicolson scheme for each time step we must solve $M$ nonlinear algebraic equations to find $M$ unknowns for fixed values of $\varepsilon$ and $\beta$. We solve that system using Newton's method.

\section{Discussion of results}

We use the numerical procedures described in Section 3 to compute the solutions for various values of $\beta$ and $\varepsilon$. In most of the computations $E=0.11$ and $M=400$. We ran the calculations with different values of $E$ and $M$ and observed that the results are independent of $E$ and $M$ to graphical accuracy.

In numerical calculations, we take all quantities in dimensionless form, so that we obtain the corresponding physical quantities by multiplying lengths by $x_{0}$ and time by $t_{0}$. Table 1 shows the range of the parameters, namely $\varepsilon$ and $\beta$, space and time increments $\Delta x$ and $\Delta t$ respectively, and the numerical methods.

We take the uniform solution as an initial guess since it satisfies not only the given differential equation but also the boundary and initial conditions.

The exact solution of the foam drainage equation has not yet been obtained, as far as we are aware. No tables were given by Cox et al. [7] or Weaire et al. [20, Page L219, Figure 6], but a comparison with their graphs shows good agreement.

In our calculations, the convergence tolerance was $10^{-8}$, that is, we terminated the iterative procedure when successive approximations agreed up to the eighth decimal place.

In Figure 1, at the left-hand side (top of the foam) the liquid fraction tends to a constant, corresponding to a wet foam. At the bottom, the liquid fraction is almost zero, corresponding to a dry foam.

We observe that the error in the Crank-Nicolson scheme has a quadratic convergence rate, and in Method I it has a linear convergence rate (see Figure 2). In Figure 1, it took 14000 iterations to reach the equilibrium solution. However, 


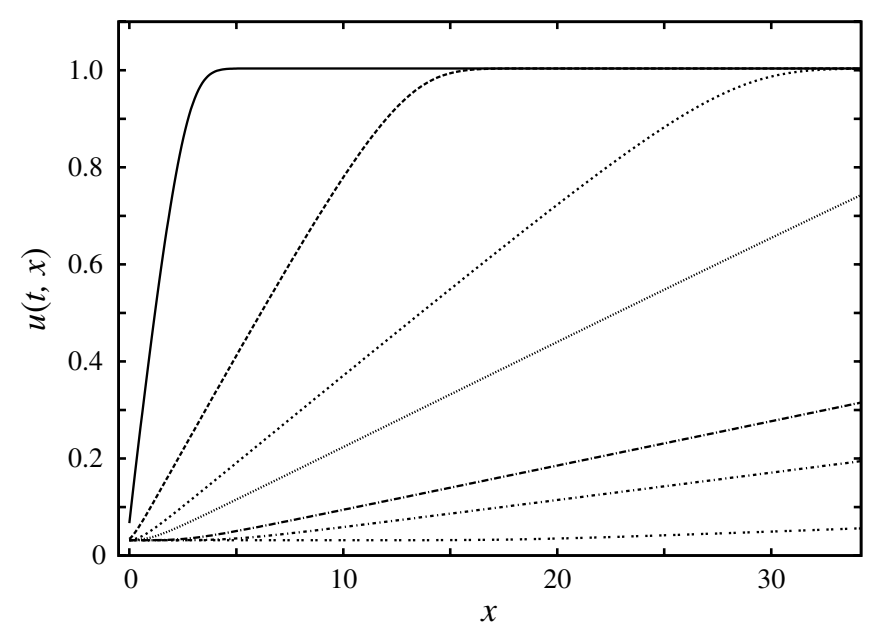

FIGURE 1. $u(t, x)$ versus $x$ plotted using the Crank-Nicolson method: $\varepsilon=0.01, \beta=0.001, \Delta x=0.0873$ and $\Delta t=0.0873$, for different times $t=5,25,55,91.6,218.2,353.5$ and 1222.3. The leftmost curve shows the solution at $t=5$. As time progresses, the curves move from left to right.

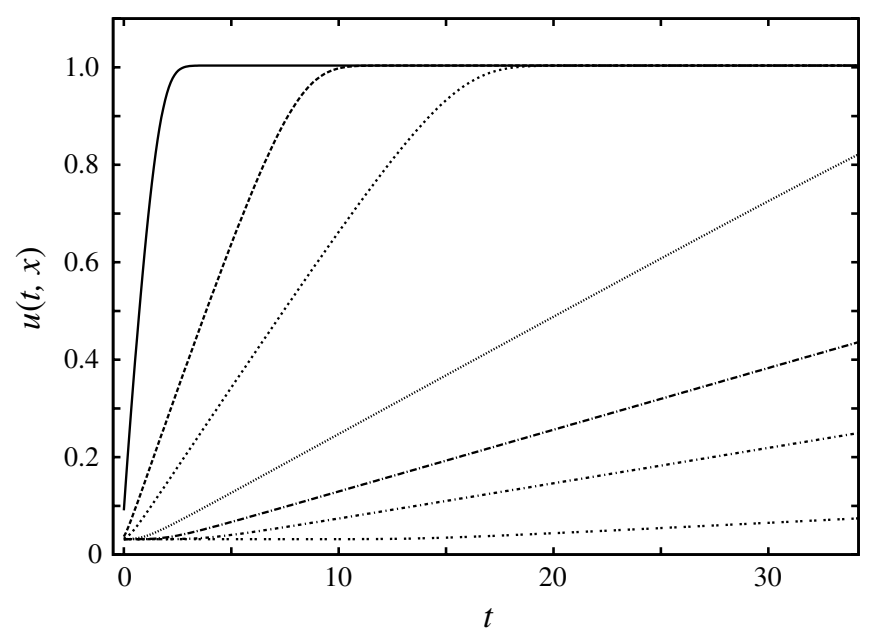

FIGURE 2. $u(t, x)$ versus $x$ plotted using Method I (implicit method): $\varepsilon=0.01, \beta=0.001, \Delta x=0.0873$ and $\Delta t=0.1$, for different times $t=5,25,48,130,250,436$ and 1450 . The leftmost curve shows the solution at $t=5$. As time progresses, the curves move from left to right.

in Figure 2 (using Method I), $\Delta t=0.0873$ was too small to reach the equilibrium solution so $\triangle t$ was taken as 0.1 . The problem with Method I is that it is only firstorder accurate in the time step and second-order accurate in space. On the other hand, the Crank-Nicolson method is second-order accurate in time and space. Notice that both methods are unconditionally stable. 


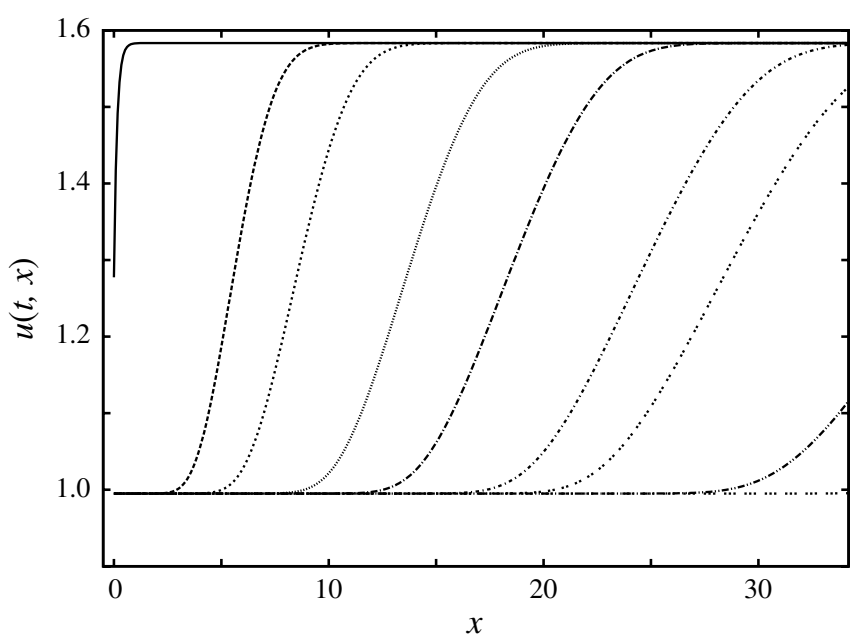

FiguRE 3. $u(t, x)$ versus $x$ plotted using the Crank-Nicolson method: $\varepsilon=0.01, \beta=0.99, \triangle t=0.0873$ and $\Delta x=0.0873$, for different times $t=0.0873$ (at the end of the first step) and then $t=5.1,7.7,12.3$, $16.5,21.9,25.4,34.1$ and 42.8 . The leftmost curve shows the solution at $t=0.0873$. As time progresses, the curves move from left to right.

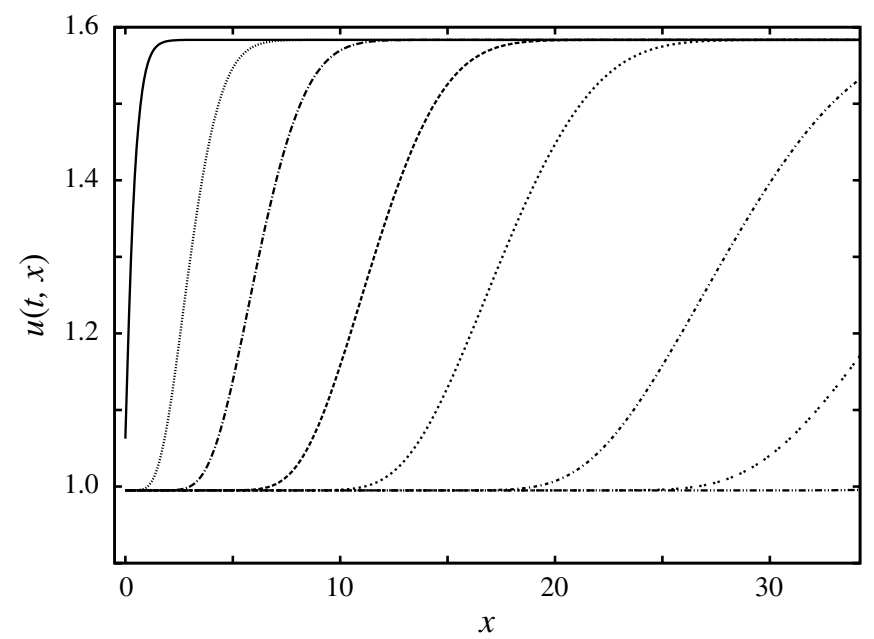

FIGURE 4. $u(t, x)$ versus $x$ plotted using Method I (implicit method): $\varepsilon=0.01, \beta=0.99, \triangle x=0.0873$ and $\Delta t=0.0873$ for different times $t=0.0873$ (at the end of the first step), 1.3, 2.7, 5, 7.6, 12.2, 16.4 and 21.8. The leftmost curve shows the solution at $t=0.0873$. As time progresses, the curves move from left to right.

The foam drainage equations have a travelling wave solution. In Figures 3, 4 and 7 the liquid fraction looks like a solitary wave, which is a wave of constant profile, moving through the foam with constant velocity. However, eventually all cases reach an equilibrium solution. 


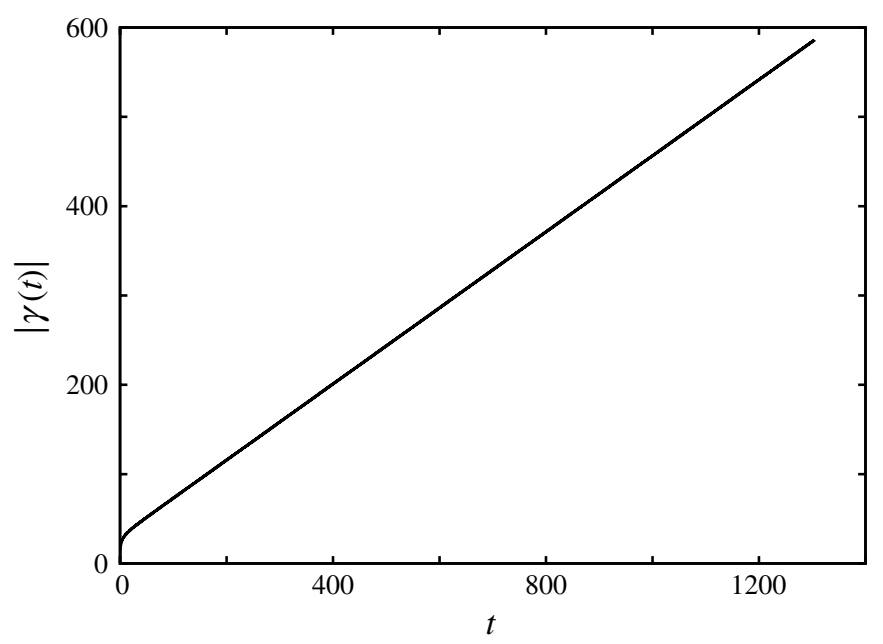

FiguRE 5. $|\gamma(t)|$ versus $t$ shown using the Crank-Nicolson method: $\varepsilon=0.01, \beta=0.001, \triangle x=0.0873$ and $\triangle t=0.0873$.

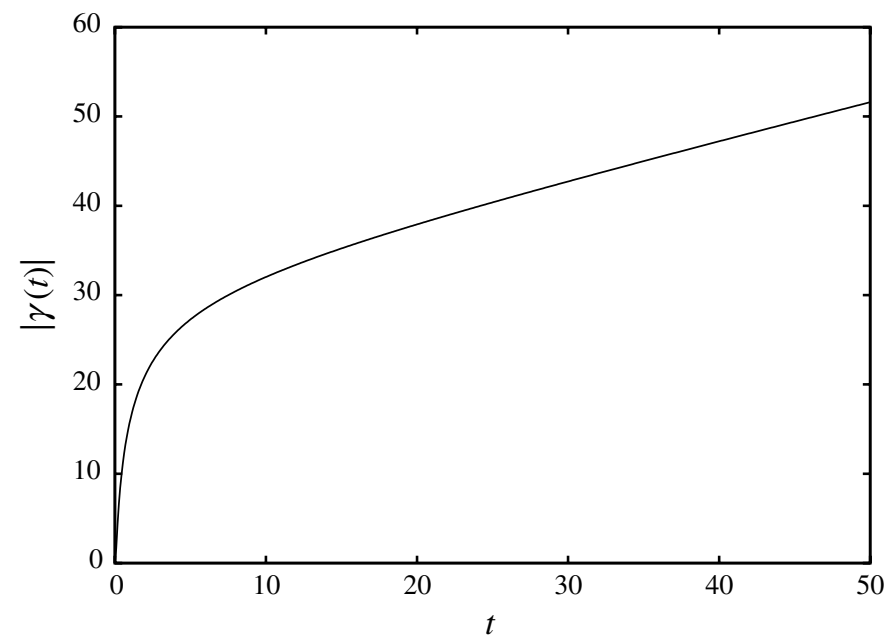

FIgURE 6. Zoomed version of $|\gamma(t)|$ versus $t$ shown using the Crank-Nicolson method: $\varepsilon=0.01$, $\beta=0.001, \triangle x=0.0873$ and $\triangle t=0.0873$.

4.1. The position of the breaking front: $\boldsymbol{\gamma}(\boldsymbol{t})$ In Section 2 we described $\gamma(t)$ as the position of the breaking front. The foam breaks when the area of the Plateau borders reaches a critical value $\beta>0$. The boundary condition at $x=\gamma(t)$ is $u(t, \gamma(t))=\beta$. The function $\gamma(t)$ is computed from $\dot{\gamma}(t)$, that is,

$$
\gamma(t)=\int_{0}^{t} \frac{d \gamma(s)}{d s} d s
$$




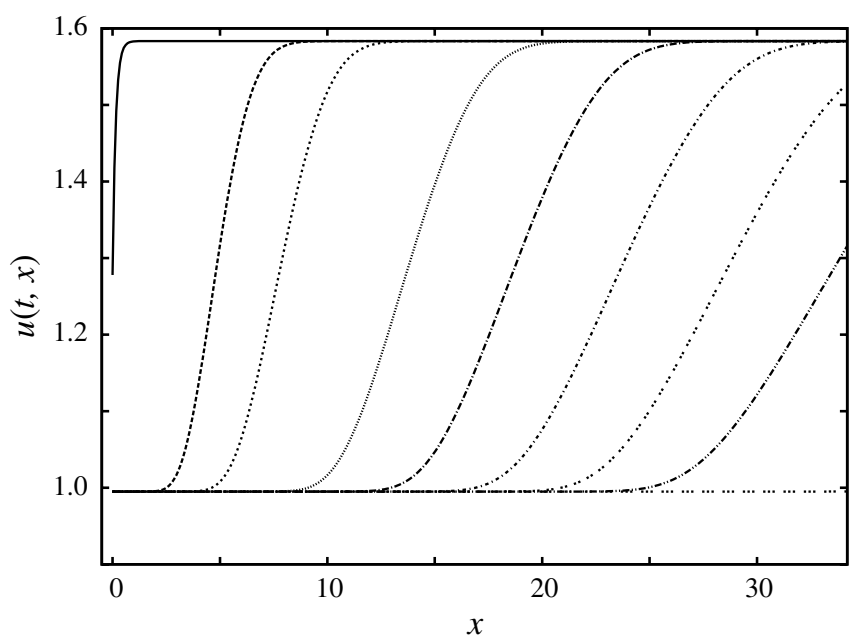

FiguRE 7. $u(t, x)$ versus $x$ plotted using the Crank-Nicolson method: $\varepsilon=10^{-7}, \beta=0.99, \triangle x=0.0873$ and $\Delta t=0.0873$ for different times $t=0.0873$ (at the end of the first step), $t=4.4,7,12.3,16.6,21$, 25.4, 29.7 and 42.8. The leftmost curve shows the solution at $t=0.0873$. As time progresses, the curves move from left to right.

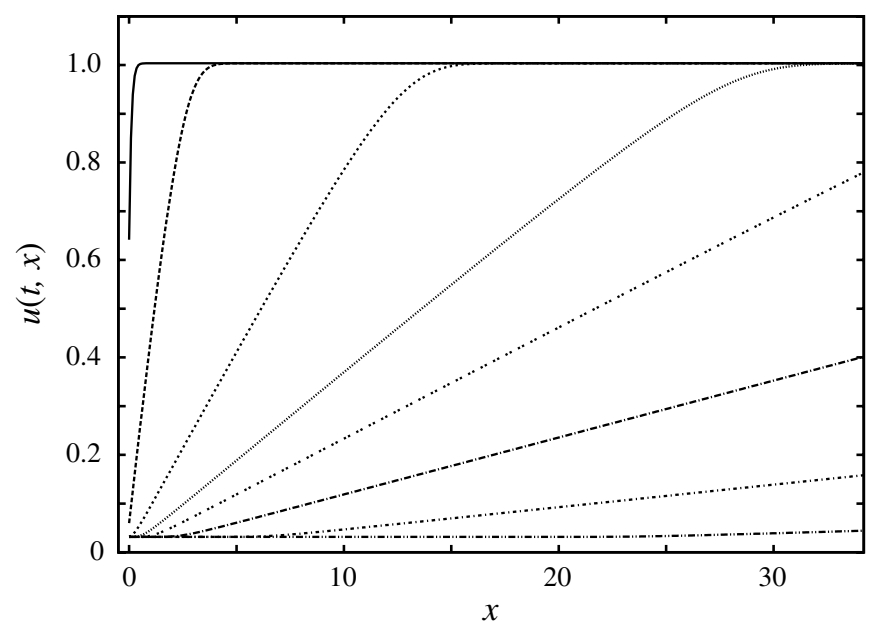

FiguRE 8. $u(t, x)$ versus $x$ plotted using the Crank-Nicolson method: $\varepsilon=10^{-7}, \beta=0.001, \triangle x=$ 0.0873 and $\Delta t=0.0873$ for different times $t=5,25,55,87.3,171.1,433$ and 1527.8. The leftmost curve shows the solution at $t=5$. As time progresses, the curves move from left to right.

As explained in Colin and Fabrie [5], the conservation of the quantity of water at the breaking front gives us the description of $\dot{\gamma}(t)$, given in Equation (2.4). We calculate the above integral by applying the trapezoidal rule.

The values of $\gamma(t)$ are positive and the curves of $\gamma(t)$ behave like a linear function except for $\varepsilon=0.01$ and $0 \leq \beta \leq 0.2$. Our calculation with $\beta$ in the range $0 \leq \beta \leq 0.2$ 
shows that $\gamma(t)$ becomes negative. One reason might be that $\dot{\gamma}(t)$ in Equation (3.2) is negative for small values of $\beta$. The profiles corresponding to this case are shown in Figure 5 (the absolute value of $\gamma(t)$ ) and enlarged in Figure 6.

4.2. Special case: $\varepsilon=10^{-7}$, Burgers' equation Notice that the general foam drainage Equation (2.5) reduces to an equation similar to the well-known inviscid Burgers' equation if $\varepsilon$ goes to zero; see Debnath [9]. The two equations are not equal, since the inviscid Burgers' equation has the form

$$
\frac{\partial u}{\partial t}+\frac{1}{2} \frac{\partial u^{2}}{\partial x}=0
$$

including the factor $1 / 2$. In Equation (2.6), $\varepsilon$ is taken to be $10^{-7}$ to compare with Burgers' equation (see Figures 7 and 8). In both cases, $\gamma(t)$ behaves like a linear function.

\section{Conclusion}

We have solved numerically the equations of a one-dimensional model of the evolution of a foam using both an implicit method and the Crank-Nicolson method in combination with Newton's method. The corresponding problem in two dimensions is left for future work.

\section{Acknowledgements}

The author is grateful for suggestions and comments made by the referee and thanks Professor Günter Bärwolff at the Technical University of Berlin for his constructive comments and proof reading.

\section{References}

[1] R. A. Adams, Sobolev spaces (Academic Press, New York, 1975).

[2] J. Banhart and D. Weaire, "On the road again: metal foams find favor", Phys. Today (2002).

[3] K. A. Brakke, "The surface evolver", Experiment. Math. 1 (1992) 141-165.

[4] T. Colin and P. Fabrie, "Semidiscretization in time for nonlinear Schrodinger-waves equations", Discrete. Contin. Dyn. Syst. 4 (1998) 671-690.

[5] T. Colin and P. Fabrie, "A free boundary problem modeling a foam drainage", Math. Models Methods Appl. Sci. 10 (2000) 945-961.

[6] S. J. Cox, G. Bradley, S. Hutzler and D. Weaire, "Vertex corrections in the theory of foam drainage", J. Phys.: Condens. Matter 13 (2001) 4863-4869.

[7] S. J. Cox, D. Weaire, S. Hutzler, J. Murphy, R. Phelan and G. Verbist, "Applications and generalizations of the foam drainage equation", Proc. R. Soc. Lond. A 456 (2000) 2441-2464.

[8] Z. Dahmani, M. M. Mesmoudi and R. Bebbouchi, "The foam drainage equation with time- and space-fractional derivatives solved by the Adomian method", Electron. J. Qual. Theory Differ. Equ. 30 (2008) 1-10.

[9] L. Debnath, Nonlinear partial differential equations (Birkhäuser, Boston, 1997).

[10] M. Durand and D. Langevin, "Physicochemical approach to the theory of foam drainage", Eur. Phys. J. E 7 (2002) 35-44. 
[11] D. Exerowa and P. M. Kruglyakov, Foam and foam films: theory, experiment and application (Elsevier, Amsterdam, 1998).

[12] I. I. Gol'dfarb, K. B. Kann and I. R. Shreiber, "Liquid flow in foams", Transactions of USSR Academy of Sciences: Ser. Mech. Liquids Gas, Fluid Dynamics 23 (1988) 244-249. [English translation], http://dx.doi.org/10.1007/BF01051894.

[13] S. A. Koehler, S. Hilgenfeldt and H. A. Stone, "A generalized view of foam drainage: experiment and theory", Langmuir 16 (2000) 6327-6341.

[14] S. A. Koehler, H. A. Stone, M. P. Brenner and J. Eggers, "Dynamics of foam: drainage", Phys. Rev. E 58 (1998) 2097-2106.

[15] A. M. Kraynik, "Foam drainage", Sandia report, 1983, SAND83-0844.

[16] P. M. Kruglyakov, S. I. Karakashev, A. V. Nguyen and N. G. Vilkova, "Foam drainage", Curr. Opinion in Colloid Int. Sci. 13 (2008) 163-170.

[17] R. A. Leonard and R. Lemlich, "A study of interstitial liquid flow in foam", A. I. Chem. Eng. J. 11 (1965) 18-25.

[18] G. Verbist, D. Weaire and A. M. Kraynik, "The foam drainage equation”, J. Phys.: Condens. Matter 8 (1996) 3715-3731.

[19] D. Weaire, "Foam physics", Adv. Eng. Mater. 4 (2002) 723.

[20] D. Weaire, S. Findlay and G. Verbist, "Measurement of foam drainage using AC conductivity", J. Phys.: Condens. Matter 7 (1995) L217-L222.

[21] D. Weaire and S. Hutzler, The physics of foams (Clarendon Press, Oxford, 1999).

[22] D. Weaire, S. Hutzler, S. Cox, N. Kern, M. D. Alonso and W. Drenckhan, "The fluid dynamics of foam", J. Phys.: Condens. Matter 15 (2003) S65-S73. 